\title{
Study on the Kinematic characteristics of different level jumpers squatting and jumping under different ground
}

Hua Hewen ( $\square$ hhw1126@zjnu.edu.cn )

Zhejiang Normal University, Jinhua, Zhejiang, China 321004

\section{Research Article}

Keywords: Countermovement jump, different ground, Kinematic

Posted Date: May 12th, 2021

DOl: https://doi.org/10.21203/rs.3.rs-506133/v1

License: (1) (1) This work is licensed under a Creative Commons Attribution 4.0 International License.

Read Full License 


\section{Abstract}

Objective: The purpose of this study is to compare the kinematic differences of different level jumping athletes in the process of squatting on different ground, and to reveal the biomechanical mechanism of the effect of squatting on different ground on training effect.

Methods: The subjects were 11 first-class and above jumping athletes (excellent group) and 11 secondclass athletes (ordinary group). The kinematic data were photographed by SONYDCRHC52E high-speed camera with a sampling frequency of 50 frames per second, and the data were analyzed by SIMI-Motion three-dimensional motion analysis system. The subjects squatted and jumped on four kinds of ground (force platform, balance pad, gymnastics pad and sand) with their hands on their hips.

Results: In the intra-group analysis, it was found that the athletes of different levels squatted and jumped on four different kinds of ground, but there was no significant difference only on the balance pad and gymnastics mat (excellent group $p=0.344$, ordinary group $p=1$ ). In the inter-group analysis, it was found that there were significant differences between different levels of athletes in balance pad $(p=0.043)$ and sand $(p=0.02)$. In the buffering stage of squatting jump, there was no significant difference in the buffering angle displacement of lower limb joints of different athletes on different ground. In the stage of pedal and extension, the angular displacement of ankle on the middle platform of the excellent group was significantly different from that of the balance pad $(p=0.014)$, gymnastics pad $(p=0.017)$ and sand $(p=0.013)$, but in the ordinary group, the ankle angular displacement of the middle platform was only significantly different from that of the gymnastics pad $(p=0.048)$ and sand $(p=0.017)$, but not significantly different from that of the balance pad $(p=0.166)$. The maximum angular velocity of hip joint of athletes of different levels was significantly different among different ground groups. The maximum angular velocity of ankle joint of common group was significantly different in force platform and sand $(p=0.032)$, gymnastics mat and sand $(p=0.008)$, but there was no significant difference in balance pad.

Conclusion: Different places have great influence on the ordinary group, and on the ground with lower elastic coefficient, the maximum angular velocity of the ankle joint in the ordinary group is much smaller than that in the excellent group, which indicates that the rapid contractile ability of ankle muscles in the ordinary group is poor on the ground with low elastic coefficient. When squatting and jumping on the ground with lower elastic coefficient, the excellent group has better body control and coordination and lower joint energy loss, which makes the jumping effect better, while the ordinary group squats and jumps on the ground with low elastic coefficient and unstable ground. the jumping effect is poor.

\section{Introduction}

Jumping technique is very important in sports, such as volleyball blocking, high jump, long jump and so on, while squat jump is usually used to evaluate the muscle strength, explosive power and rapid development of lower limbs. Squat jump needs to quickly do the action of squatting down, and then quickly jump upward, and through the stretch reflection mechanism, elastic energy storage and release to 
reveal the characteristics of lengthening and shortening cycle ${ }^{[3]}$. During the period of lengthening and shortening the cycle, the elastic energy stored during the eccentric phase (i.e. stretching) is used to cause the pre-activated muscle to elongate rapidly (the eccentric phase), and then the shortening contraction begins immediately (the centripetal phase). Compared with the centripetal contraction alone, this centrifugal / centripetal contraction mechanism produces a greater contractile force ${ }^{[4]}$. As a typical exercise of lengthening and shortening periods, squat jumping is usually used to measure jumping ability in the field of sports training and research. In the squat jump test, the external factors that affect the squat jump test are easy to control, so many researchers analyze the movement through the characteristics of biomechanical and muscle mechanical parameters of human movement. Especially in the detection and evaluation of lower limb muscle strength and explosive force, and the utilization rate of elastic potential energy, the value of squat jump test is particularly important. Squat jumping is not only an index to evaluate the explosive power of athletes' lower limbs, but also one of the commonly used methods of lower limb strength training and bouncing training in the process of sports training.

In sports, sports performance depends on speed, agility and strength [8], while athletes training in different environments will have different effects. Differences in sports surface components will affect athletes' speed, agility and strength ${ }^{[10]}$. When athletes train on different sports surfaces, the athletes will have different reactions because of the different surface characteristics. for example, the different physical structure between the natural turf surface and the artificial turf surface will make the human body change the direction of the force in the jumping process, thus changing the sports ability. Meyers et al. ${ }^{[11]}$ found that athletes on traditional artificial turf surfaces can run faster and change direction faster than natural turf surfaces. However, when athletes train on different ground, how the different characteristics of the surface have an impact on people still need further research, so that athletes can get as much training benefit as possible in the shortest possible time ${ }^{[12]}$. Choosing a suitable ground for squatting and jumping training is one of the key factors that affect the effect of lower limb strength training. With the training of athletes on different materials and jumping training on different floors, the negative effects are gradually highlighted. Two of them are the problems that researchers and coaches pay most attention to: first, when squatting and jumping training on hard ground, the ground will have a high impact on the joints at the moment of landing. Easily lead to bone and ligament injury ${ }^{[13]}$. Second, in some events, hard underground squatting training can not effectively improve the special sports performance of athletes, and the effect of sports transfer is not significant ${ }^{[14.15]}$. In order to solve these problems, coaches and researchers are constantly exploring new solutions to reduce the negative impact of squat and jump training of different materials. In practice, coaches arrange athletes to squat and jump in various environments, such as sand, cushion, hard ground, trampoline, water and so on ${ }^{[16-20]}$. At the same time, a large number of studies have compared the squatting and jumping training of different materials, but the results are not consistent. There is a great controversy as to whether the squatting and jumping training of different materials can improve the training efficiency. Different surface training will affect the performance, speed and power of the lower limb joints of the body. In the process of jumping, how the movement surface affects the lower limb joints and the influence of the movement surface on the lower limb joints is not clear. The purpose of this study is to compare the differences of lower limb 
joint kinematics of different level jumping athletes in the process of squatting and jumping on different sports surfaces.

\section{Research Methods}

\subsection{Object of study}

The experiment selection 11 (excellent groups) and 11th athletes (excellent group) and 11 (ordinary groups) of the three levels of sports colleges, the Sports Institute of Zhejiang Normal University (ordinary group), and participate in this study, physical health, no exercise damage, sports ability Normal, recent diseases, no neuromblex, cardiopulmonary function, have a good rest before testing, the muscles have no fatigue, all subjects have filled out the informed consent, and the experiment has obtained the Zhejiang Normal University Laboratories. Approval, the basic information of the subject is shown in Table 1:

\section{Table 1 basic information of subjects}

\begin{tabular}{|lllll|}
\hline Group & $\mathrm{N}$ & Age & Height $(\mathrm{cm})$ & Body weight(kg) \\
\hline Excellent group & 11 & $20.8 \pm 2.0$ & $175 \pm 4.0$ & $65.2 \pm 5.0$ \\
\hline General group & 11 & $20.8 \pm 2.0$ & $175 \pm 4.0$ & $65.2 \pm 5.0$ \\
\hline
\end{tabular}

\subsection{Experimental flow}

$\nabla_{1} \otimes$ Before this experiment, different ground recovery coefficients are tested, the test method is the sphere rebound height method, and the standard to evaluate the motion surface recovery coefficient is the sports surface test index-sphere rebound height (ASTMF2117-2010) issued by the International Association for material testing ((ASTM)). Tennis balls are used on these four sports surfaces to let tennis balls fall freely from a height of 2 meters, and each surface falls 3 times. The recovery coefficient of the sports surface is evaluated by calculating the average rebound height of tennis balls after falling 3 times. The higher the springback height, the greater the recovery coefficient of the sports surface. The elastic coefficients of floors of different materials are calculated by the formula: $\mathbb{H b}$ is the bounce height of the ball and hd is the falling height of the ball. Finally, it is concluded that the recovery coefficients of the four kinds of ground are force platform $>$ balance pad $>$ gymnastics pad $>$ sand.

$\nabla 2 \varangle$ Before the experiment, the subjects were asked to warm up and jog on the treadmill at the speed of $5 \mathrm{~km} / \mathrm{h}$ for 10 minutes, followed by 5 min dynamic stretching to move the joints and muscles of the body, especially the joints of the lower limbs, to prevent the subjects from being injured during the experiment.

$\triangle 3 \otimes T$ The staff counted the basic conditions of the subjects, such as height, weight, age, lower limb length, thigh length, calf length, foot length, medical history and so on, affixed reflective markers on the subjects, and informed the subjects of the whole experimental process. make the subjects familiar with the whole process of the experiment. 
\4囚In the experiment, the whole movement process of squatting jump was photographed (sampling frequency 50 frames per second). The exposure time was set and placed vertically in the positive direction of the subjects, the distance was about $5 \mathrm{~m}$. Before the test, the subjects first fixed the PEAK three-dimensional calibration frame (the frame coordinate $X$ represents the left and right direction, $Y$ represents the front and rear direction, $Z$ represents the vertical direction) to calibrate the range of motion of the subjects. After the calibration is completed, the positions of the two cameras and various shooting conditions remain unchanged, and the distinction is suspended for each test, and the shooting frequency is 50 frames per second. At the beginning of the exercise, the frame was removed to photograph the subjects' whole squatting and jumping process. In order to accurately observe the motion track of the main joints of the human body during video analysis, reflective markers were affixed to the center of the main joints of the subjects (see Table 2).

\section{Table 2 name and fixed position of marked points}

\begin{tabular}{|llc|}
\hline Dimension point name & Position & Number \\
\hline Head & Left and right temples & 2 \\
\hline Left and right shoulder & Acromion & 2 \\
\hline Left and right elbows & Lateral epicondyle of humerus & 2 \\
\hline Left and right wrist & $\begin{array}{l}\text { The midpoint of the line between the ulna and the styloid } \\
\text { process of the radius }\end{array}$ & 2 \\
\hline $\begin{array}{l}\text { Left and right anterior } \\
\text { superior iliac spine }\end{array}$ & The highest point of the great rotor & 2 \\
\hline $\begin{array}{l}\text { Left and right outer knee } \\
\text { Left and right lateral } \\
\text { malleolus }\end{array}$ & $\begin{array}{l}\text { Lateral condyle of femur } \\
\text { Lateral malleolus of fibula }\end{array}$ & 2 \\
\hline Cusp of left and right foot & $\begin{array}{l}\text { Between the second and third metatarsals of the foot, the } \\
\text { same height as the root bone. }\end{array}$ & 2 \\
\hline
\end{tabular}

(5) Use the SonyDCRHC52E high-speed camera and Kistler dynamic system to collect kinematics and kinetics during completion of the squat. Subjects have completed the squat jump in different materials on the laboratory platform, and in the laboratory with three-dimensional performance platform, the angle between the main optical axes of the two camera is about 90 degrees, and 2 sets. SONYDCRHC52E digital camera (sampling frequency 25 frames / sec), camera effective shooting range is about $5 \mathrm{~m}$ long, $2.5 \mathrm{~m}$ wide and $2.5 \mathrm{~m}$ high. Within the effective shooting range, a Swiss Kistler dynastucting station (contact area $90 * 60 * 10 \mathrm{~cm}$ ) is placed on the ground, and the sampling frequency is $1000 \mathrm{~Hz}$ (Fig. 1).

The subject stands on the hands of the hands on the hands of the hands, the double-footed is more width. When listening to the password of the staff, in turn, squat three times in four terrestrials, four 
terrestrial tests in the same The day is completed, and each ground test is $10 \mathrm{~s}$, each ground test interval is $1 \mathrm{~min}$.

\subsection{Statistical analysis}

Using Excel, SPSS21.0 data processing software sorted the effective data taken, the subject 3 test data takes the average of 3 test data for each floor after data processing, all measurement parameters The data is expressed in average $(x) \pm$ standard deviation (SD); the difference in the kneeling of the lower extremities during different levels of different levels of different levels of different levels of different levels of different levels, significant comparison Method, significant level $\mathrm{P}<0.05$.

\section{Results}

\subsection{Jumping height}

As can be seen from Figure 1, the difference between different levels of athletes in force, balance pad, gymnastic pad, and sand is found using repeated measurement variance analysis. Different level athletes are discovered under four different grounds in the group analysis. Jump, there is no significant difference in balance mat and gymnastics (excellent group $P=0.344$, ordinary group $P=1$ ); discovery different level athletes in balance pad $(P=0.043)$ and sand $(p=0.02)$ Differences were significant.

\subsection{Hip, knee, ankle displacement}

From Fig. 2 and Table 3, it is known that the cushioning angle displacement of different athletes in different plants in different plants is not significant difference in different plants $(P>0.05)$; in Fig. 3 and Table 4 Excellent group and ordinary group There is no significant difference in the knee angle displacement group ( $P>0.05)$, but the hip angular displacement of different athletes in the gymnastics is subjected to a significant difference $(P=0.045)$, and the difference between groups), excellent group The ankle angular displacement on the tower is significantly different from the balance pad $(P=0.014)$, gymnastics pad $(p=0.017)$ and sand $(P=0.013)$, but found in ordinary groups The joint angular displacement is only significantly different from the gym $(P=0.048)$ and sand $(P=0.017)$, and there is no significant difference in the balance pad $(P=0.166)$.

Table 3 Different horizontal athletes with different ground hips, knee, ankle buffer angle displacement 


\begin{tabular}{|c|c|c|c|c|c|}
\hline & & Excellent group & Ordinary group & $\mathrm{F}$ & $P$ \\
\hline \multirow[t]{4}{*}{ Hip joint } & Force & $93.79 \pm 15.02$ & $82.89 \pm 17.32$ & 2.483 & 0.131 \\
\hline & Balance pad & $88.89 \pm 18.88$ & $80.53 \pm 18.45$ & 1.105 & 0.306 \\
\hline & Gym & $93.66 \pm 17.19$ & $80.04 \pm 20.58$ & 2.837 & 0.108 \\
\hline & Sand & 99.0018 .67 & $84.40 \pm 23.85$ & 2.558 & 0.125 \\
\hline \multirow[t]{4}{*}{ Knee } & Force & $78.06 \pm 9.65$ & $71.11 \pm 10.29$ & 2.669 & 0.118 \\
\hline & Balance pad & $76.01 \pm 6.36$ & $72.63 \pm 11.71$ & 0.708 & 0.41 \\
\hline & Gym & $77.43 \pm 10.34$ & $71.25 \pm 13.09$ & 1.514 & 0.233 \\
\hline & Sand & $83.90 \pm 14.82$ & $74.83 \pm 14.90$ & 2.052 & 0.167 \\
\hline \multirow[t]{4}{*}{ Ankle joint } & Force & $32.37 \pm 5.33$ & $33.35 \pm 5.95$ & 0.166 & 0.688 \\
\hline & Balance pad & $32.39 \pm 7.04$ & $33.93 \pm 7.48$ & 0.246 & 0.625 \\
\hline & Gym & $33.20 \pm 7.40$ & $33.44 \pm 8.95$ & 0.005 & 0.945 \\
\hline & Sand & $36.52 \pm 7.27$ & $35.15 \pm 6.92$ & 0.203 & 0.657 \\
\hline
\end{tabular}

Table 4 Different horizontal athletes with different ground hips, knee, and ankle

\begin{tabular}{|llllll|}
\hline & & Excellent group & Ordinary group & $F$ & $P$ \\
\hline Hip joint & Force & $93.36 \pm 11.88$ & $84.90 \pm 13.97$ & 2.339 & 0.142 \\
& Balance pad & $89.58 \pm 15.26$ & $81.49 \pm 15.33$ & 1.54 & 0.229 \\
& Gym & $94.44 \pm 11.66$ & $81.04 \pm 17.18$ & 4.586 & $0.045^{*}$ \\
& Sand & $96.93 \pm 18.53$ & $86.16 \pm 25.76$ & 1.268 & 0.273 \\
\hline Knee & Force & $86.48 \pm 7.0$ & $82.61 \pm 12.79$ & 0.776 & 0.389 \\
\hline & Balance pad & $84.26 \pm 9.05$ & $79.60 \pm 10.27$ & 1.272 & 0.273 \\
\hline & Gym & $88.40 \pm 7.56$ & $81.69 \pm 12.93$ & 2.203 & 0.153 \\
\hline & Sand & $93.91 \pm 12.08$ & $87.86 \pm 15.72$ & 1.023 & 0.324 \\
\hline & Force & $45.44 \pm 9.19^{*}$ & $45.92 \pm 7.05^{*}$ & 0.029 & 0.865 \\
\hline & Balance pad & $50.96 \pm 5.83^{*}$ & $49.63 \pm 6.36$ & 0.262 & 0.615 \\
\hline & Gym & $51.55 \pm 3.9^{*}$ & $51.22 \pm 6.36^{*}$ & 0.022 & 0.883 \\
\hline & Sand & $53.44 \pm 4.36^{*}$ & $53.65 \pm 5.97^{*}$ & 0.009 & 0.924 \\
\hline
\end{tabular}


* Indicates a significant difference $P<0.05$

\subsection{Hip, knee, big angle velocity}

By analyzing different levels of athletes in different ground hips, knee, and ankle, the maximum angular velocity of different levels at different plants in different plants is not significant difference in the group, but in the analysis of the maximum angular velocity of knee joint buffers. Different levels of athletes have significant differences between BRT $(P=0.016)$ and sand $(P=0.02)$ group (Fig. 4 and Table 5); can be seen from Figures 5 and Table 6, different levels of athletes are the largest in the stretch stage The angular velocity is significantly different from the balance pad $(P=0.007)$, the gym $(p=0.003)$ and the sand $(P=0.029)$ group difference, and the maximum angular velocity of the ankle joint of different athletes is discovered. The ordinary group has a significant difference between the maximum angular velocity group of the ankle joint of the gymptom pad $(P=0.015)$, but there is no significant difference in the ankle joints when performing the jump on different places, and analyzes the regular group The ordinary group is in the equilibrium pads in the balanced pad in the equilibrium pads in the balance mat.

\section{Table 5 Different horizontal athletes with different ground hips, knee, ankle buffer maximum angular velocity}

\begin{tabular}{|llllll|}
\hline & & Excellent group & Ordinary group & $F$ & $P$ \\
\hline Hip joint & Force & $485.14 \pm 76.37$ & $451.49 \pm 67.66$ & 1.196 & 0.287 \\
& Balance pad & $474.00 \pm 87.52$ & $428.45 \pm 113.68$ & 1.109 & 0.305 \\
& Gym & $491.73 \pm 73.63$ & $431.34 \pm 102.12$ & 2.531 & 0.127 \\
\hline Knee & Sand & $481.43 \pm 62.21$ & $443.14 \pm 107.94$ & 1.039 & 0.32 \\
\hline & Force & $521.15 \pm 66.31$ & $457.28 \pm 45.92$ & 6.898 & $0.016^{*}$ \\
\hline & Balance pad & $468.80 \pm 57.75$ & $438.57 \pm 69.88$ & 1.223 & 0.282 \\
\hline \multirow{2}{*}{ Ankle joint } & Gym & $490.47 \pm 54.63$ & $452.40 \pm 50.38$ & 2.887 & 0.105 \\
\hline & Sorce & $431.71 \pm 66.23$ & $413.51 \pm 45.04$ & 0.568 & 0.46 \\
\hline & Balance pad & $411.87 \pm 50.02$ & $394.05 \pm 41.90$ & 0.82 & 0.376 \\
\hline & Gym & $412.59 \pm 53.39$ & $392.49 \pm 69.24$ & 0.582 & 0.455 \\
\hline & Sand & $414.56 \pm 46.50$ & $377.19 \pm 58.13$ & 2.773 & 0.111 \\
\hline
\end{tabular}

* Indicates a significant difference $\mathrm{P}<0.05$

Table 6 Different horizontal athletes with different ground hips, knee, and ankle 


\begin{tabular}{|llllll|}
\hline \multirow{2}{*}{ Hip joint } & Excellent group & Ordinary group & $F$ & $P$ \\
& Force & $466.74 \pm 28.28$ & $400.39 \pm 38.10$ & 21.51 & $0.000^{*}$ \\
& Balance pad & $459.04 \pm 37.86$ & $417.68 \pm 25.36$ & 9.058 & $0.007^{*}$ \\
& Gym & $471.30 \pm 39.65$ & $406.52 \pm 51.37$ & 10.963 & $0.003^{*}$ \\
\hline Knee & Sand & $468.91 \pm 52.64$ & $399.75 \pm 82.47$ & 5.497 & $0.029^{*}$ \\
\hline & Force & $525.28 \pm 62.54$ & $526.73 \pm 59.56$ & 0.003 & 0.956 \\
\hline & Balance pad & $513.88 \pm 51.35$ & $514.10 \pm 53.37$ & 0.000 & 0.992 \\
\hline & Gym & $532.45 \pm 30.96$ & $517.11 \pm 50.68$ & 0.734 & 0.402 \\
\hline Ankle joint & Fand & $548.50 \pm 52.00$ & $533.00 \pm 60.29$ & 0.365 & 0.552 \\
\hline & Force & $554.53 \pm 60.59$ & $499.27 \pm 75.34$ & 3.576 & 0.073 \\
\hline & Balance pad & $513.85 \pm 42.90$ & $503.41 \pm 56.09$ & 0.241 & 0.629 \\
\hline & Gym & $553.07 \pm 52.14$ & $499.30 \pm 42.21$ & 7.069 & $0.015^{*}$ \\
\hline & Sand & $563.46 \pm 38.35$ & $553.20 \pm 48.17$ & 0.305 & 0.587 \\
\hline
\end{tabular}

* Indicates a significant difference $\mathrm{P}<0.05$

\section{Discussion}

This study aims to analyze different levels of athletes in different grouse exercise in different places, discovering in daily training, athletes can jump training or testing on different grounds, but what is the existence? The difference is still unclear. This study passed the height analysis of different levels of athletes on different grounds, and the lower extremity hip, knee, ankle displacement and maximum angular velocity found that different levels of athletes have significant differences in the jump height of different places. There is only differences in the stretching in terms of angular displacement and maximum angular velocity.

At present, the research on squat jump in our country is mostly focused on the influence of arm swing on take-off effect, the comparative analysis of mechanical characteristics of different sports after squat jump training and the influence of stretching action on squat jump ability before exercise. You Yonghao et al. ${ }^{[7]}$ analyzed the biomechanical characteristics of young men with or without arm swing in the form of squat jump, and mainly revealed the action mechanism of arm swing in squat jump from the aspects of longitudinal jump buffer stage, pedal extension stage and maximum pedal extension buffer time. Squat jumping is an indispensable sports skill in many sports, and the success or failure of jumping events in sports largely depends on the ability of athletes' lower limbs. This is why many studies analyze squatting movements from a physical point of view, in order to find the influencing factors that can not be 
distinguished by the naked eye in the jumping process, so as to improve the competitive ability of athletes.

Through the experiment, it is found that the surface recovery coefficient has a great influence on the jumping height, and the vertical jumping height of the human body can reflect the athletes' longitudinal jumping ability and explosive power. The recovery coefficients of four kinds of ground are tested before the experiment, and the results are force platform $>$ balance pad $>$ gymnastics pad $>$ sand. Previous studies have found that the greater the surface recovery coefficient, the higher the jump height, but in the test of the ordinary group, the height of jumping on the balance pad in the ordinary group is lower than that on the gymnastics pad. The reason may be that the instability of the balance pad makes the athletes lose more energy during take-off. However, this situation did not occur in the excellent group, which may be due to the better physical control and coordination ability of the elite group athletes, and less energy loss during the jump. When jumping on the unstable balance pad, the ground will deform to a certain extent in the pedal and extension stage, which makes it difficult for the force of the joint in the final exertion stage to advance along the vertical direction, and finally leads to the decrease of the jump height. It has also been reported that jumping in the sand absorbs almost $100 \%$ of the energy generated by the impact ${ }^{[23]}$. Therefore, it is likely that the energy generated by the muscles in the jump buffer stage has a great effect on the squat jump, so the surface reaction force is small and the jump height is low. The characteristics of energy absorption in the sand will increase the contraction time of the lower limb muscles of the squatting jump and excite the leg extensor before contraction. therefore, the squatting jump in the sand does more work than on the hard ground. Some studies have found that the squat height $(28.8 \mathrm{~cm})$ on the hard surface is significantly higher than that on the sand $(24.8 \mathrm{~cm})$, which may be related to the instability and low stiffness of the sand surface, which reduces the take-off speed of squatting jump ${ }^{[24]}$.

The angular displacement of different level athletes on different ground is shown in figure 2 and figure 3 . The angular displacement of lower limb joint of elite athletes is higher than that of ordinary athletes, and the angular displacement of lower limb joint sand is higher than that of other grounds. there was no significant difference in the angular displacement of hip joint and knee joint during the pedal and extension stage of squatting jump in figure 3 . However, when squatting and jumping on the gymnastic mat, there were significant differences in hip angular displacement among different athletes (inter-group difference). In the excellent group, the ankle angular displacement on the force platform was significantly different from that of balance pad, gymnastics pad and sand, but it was found in the ordinary group. the ankle angular displacement of the middle force platform in the ordinary group was only significantly different from that of the gymnastics pad and sand, but there was no significant difference with the balance pad. Some studies have found that when jumping on a hard surface, the range of motion of the hip and knee joint is smaller than that in the sand ${ }^{[25]}$. In addition, the study of Tilp et al. ${ }^{[26]}$ shows that the flexion of the knee joint in the pedal and extension stage of the volleyball spike is significantly higher in the sand than on the indoor wooden floor, indicating that the flexion range of the soft surface is higher than that of the hard surface. The reason for this difference is that the ground produces a higher reaction 
force during the jump, and the flexion angle of the joint increases in order to avoid skidding and maintain balance. Therefore, the range of motion of the lower limb joint will increase with the increase of the ground reaction force. Therefore, the lower limb joints on the softer ground will bend more than other surfaces, but the buckling range of sand is higher than that of other surfaces. This difference can be understood as when squatting and jumping in the sand, the mobility and instability of the sand lead to a decrease in balance, which will preserve the balance of the body by increasing the curvature of the joints of the lower extremities.

The sequence of human lower limb joint activity is as follows: the muscle groups of hip, knee and ankle should produce centripetal contraction in turn in the process of squatting and stretching, and the hip joint and knee joint of lower limbs are equipped with large muscle groups. in the early stage of squatting, jumping and stretching to overcome the greater inertia resistance of the human body mainly depends on the centripetal contraction of the large muscle group of the lower extremities. The ankle joint is mainly located in the small muscle group, the contraction force is small, but the contraction speed is faster than the large muscle group. The ankle joint can produce centripetal contraction only in the upward stage of the center of gravity of the human body and less resistance. In the squatting jump pedal extension stage, the ankle joint is the last force joint of the lower limbs off the ground, and it is also the last force moment of the pedal extension stage. In this study, when different level athletes squatted and jumped on different floors, there was basically no significant difference in the maximum angular velocity of knee joint during buffering and pedal extension, and there was no difference in the maximum angular velocity of hip joint during buffering. there was significant difference between groups during pedal and stretch. there was no difference in the maximum angular velocity of ankle joint within the excellent group, but there was difference between groups on the gymnastic mat. And there were significant differences in force platform and sandy land, gymnastic mat and sandy land in the ordinary group. The active force of the ankle joint in the final stage of pedaling and extension makes the human body push off the ground quickly, and the direction of the ankle joint is the same as the moving direction of the center of gravity, so the active and fast force of the ankle joint will make the jumping effect better. The strength of the extensor and flexion muscles of the hip joint and knee joint will affect the jumping effect, so it is necessary to strengthen the muscle strength training in the training process, and also strengthen the rapid muscle expansion training of the ankle joint.

\section{Application In Practice}

Different sports surfaces will have a great impact on the sports effect of the human body, so attention should be paid to the selection of venues in jumping training. In this experiment, it is found that the maximum angular velocity of the hip joint and knee joint of the ordinary group is smaller than that of the excellent group, indicating that the muscle strength of the lower extremities of the ordinary group is relatively weak, and the strength training of the lower limbs should be strengthened in sports training. At the same time, the maximum angular velocity of the ankle in the ordinary group is quite different in the pedal and extension stage, indicating that the rapid contractile ability of the ankle muscle in the ordinary 
group is poor, so the training of the rapid contractile ability of the ankle muscle should be strengthened on the ground with low recovery coefficient.

\section{Conclusion}

(1) Differently face the general group, and in the ground, the maximum angular velocity of the ankle joint in the ordinary group is small, indicating that the ordinary group of ankle muscles is more elastic coefficient. The low ground rapid contraction ability is poor;

(2) When the surface is extracted, the excellent group is good for physical control and coordination, and the joint energy loss is low, so that the jump effect is better; When the ordinary group is subjected to a lower elastic coefficient and unstable ground, the jump effect is poor.

\section{Declarations}

\section{Competing interests}

The authors declare no competing interests.

\section{References}

[1] Elvira J, Rodriguez I G, Riera M M, et al. Comparative study of the reliability of three jump tests with two measurement systems[J]. JOURNAL OF HUMAN MOVEMENT STUDIES. 2001(No.5): 369-383.

[2] Mclellan C P C B, Lovell D I, Gass G C. The role of rate of force development on vertical jump performance.[J]. J Strength Cond Res. 2011(No.2): 379.

[3] Bosco C, Komi P V. Utilization of stored elastic energy in leg extensor muscles by men and women[J]. Medicine and Science in Sports. 1978: 261.

[4] Loturco I, Suchomel T, Bishop C, et al. 1RM Measures or Maximum Bar-Power Output: Which is More Related to Sport Performance?[J]. International journal of sports physiology and performance. 2018: 118.

[5] Gong Jianfang, Li Shan, Wang Baofeng. Effects of different stretching forms and delay time on explosive force of lower extremities [J]. Journal of Chengdu Institute of physical Education. 2012 (6): 8387.

[6] Chtourou H, Aloui A, Hammouda O, et al. Effect of static and dynamic stretching on the diurnal variations of jump performance in soccer players[J]. PLoS One. 2013, 8(8): e70534.

[7] You Yonghao, Wen ailing, Zhang Meng, etc. Analysis of biomechanical characteristics of young men's CMJ exercise [J]. Journal of Hebei Institute of physical Education. 2015 Journal 29 (01): 77-80. 
[8] Gajewski J, Michalski R, Busko K, et al. Countermovement depth - a variable which clarifies the relationship between the maximum power output and height of a vertical jump[J]. Acta Bioeng Biomech. 2018, 20(1): 127-134.

[9] Gucluover A, Gulu M. Developing a new muscle power prediction equation through vertical jump power output in adolescent women[J]. Medicine (Baltimore). 2020, 99(25): e20882.

[10] Handel M, Horstmann T, Dickhuth $\mathrm{H} \mathrm{H}$, et al. Effects of contract-relax stretching training on muscle performance in athletes[J]. Eur J Appl Physiol Occup Physiol. 1997, 76(5): 400-408.

[11] Meyers M C. Incidence, mechanisms, and severity of game-related college football injuries on FieldTurf versus natural grass: a 3-year prospective study[J]. Am J Sports Med. 2010, 38(4): 687-697.

[12] Mao Peng. Further discussion on the overall setting [J]. Sports and science. 201637 (04): 23-25

[13] Dufek J S, Bates B T. Biomechanical factors associated with injury during landing in jump sports[J]. Sports Med. 1991, 12(5): 326-337.

[14] Kibele A, Classen C, Muehlbauer T, et al. Metastability in plyometric training on unstable surfaces: a pilot study[J]. BMC Sports Sci Med Rehabil. 2014, 6: 30.

[15] Lin Yiguan, Xu Yangyang, Huang Jianhua, et al. Research progress of rapid telescopic compound training on unstable ground [J]. Chinese Journal of Sports Medicine. 2020 (7): 577-84.

[16] Zhang Xiaohui. A new training method-super isometric training in water and its application in sports training [J]. China Sports Science and Technology. 2014 (6): 19-23

[17] Binnie M J, Dawson B, Pinnington $\mathrm{H}$, et al. Sand training: a review of current research and practical applications[J]. J Sports Sci. 2014, 32(1): 8-15.

[18] Kibele A, Classen C, Muehlbauer T, et al. Metastability in plyometric training on unstable surfaces: a pilot study[J]. BMC Sports Sci Med Rehabil. 2014, 6: 30.

[19] Crowther R G, Spinks W L, Leicht A S, et al. Kinematic responses to plyometric exercises conducted on compliant and noncompliant surfaces[J]. J Strength Cond Res. 2007, 21(2): 460-465.

[20] Prieske O, Muehlbauer T, Krueger T, et al. Role of the trunk during drop jumps on stable and unstable surfaces[J]. Eur J Appl Physiol. 2015, 115(1): 139-146.

[21] Auml K H, Kkinen, Komi P V, et al. Effect of explosive type strength training on isometric force- and relaxation-time, electromyographic and muscle fibre characteristics of leg extensor muscles[J]. Acta physiologica Scandinavica. 1985(No.4): 587-600.

[22] Bobbert M F. Drop jumping as a training method for jumping ability[J]. Sports medicine (Auckland, N.Z.). 1990(No.1): 7-22. 
[23] Bishop D. A comparison between land and sand-based tests for beach volleyball assessment[J]. Journal of sports medicine and physical fitness. 2003, 43(4): 418-423.

[24] Giatsis G, Kollias I, Panoutsakopoulos V, et al. Volleyball:Biomechanical differences in elite beachvolleyball players in vertical squat jump on rigid and sand surface[J]. Sports Biomechanics. 2004(No.1): 145-158.

[25] George Giatsis I K V P. Biomechanical Differences in Elite Beach-Volleyball Players in Vertical Squat Jump on Rigid and Sand Surface[J]. 2004, 1(3): 145-158.

[26] Tilp M, Wagner H, Muller E. Differences in 3D kinematics between volleyball and beach volleyball spike movements[J]. Sports Biomech. 2008, 7(3): 386-397.

\section{Figures}

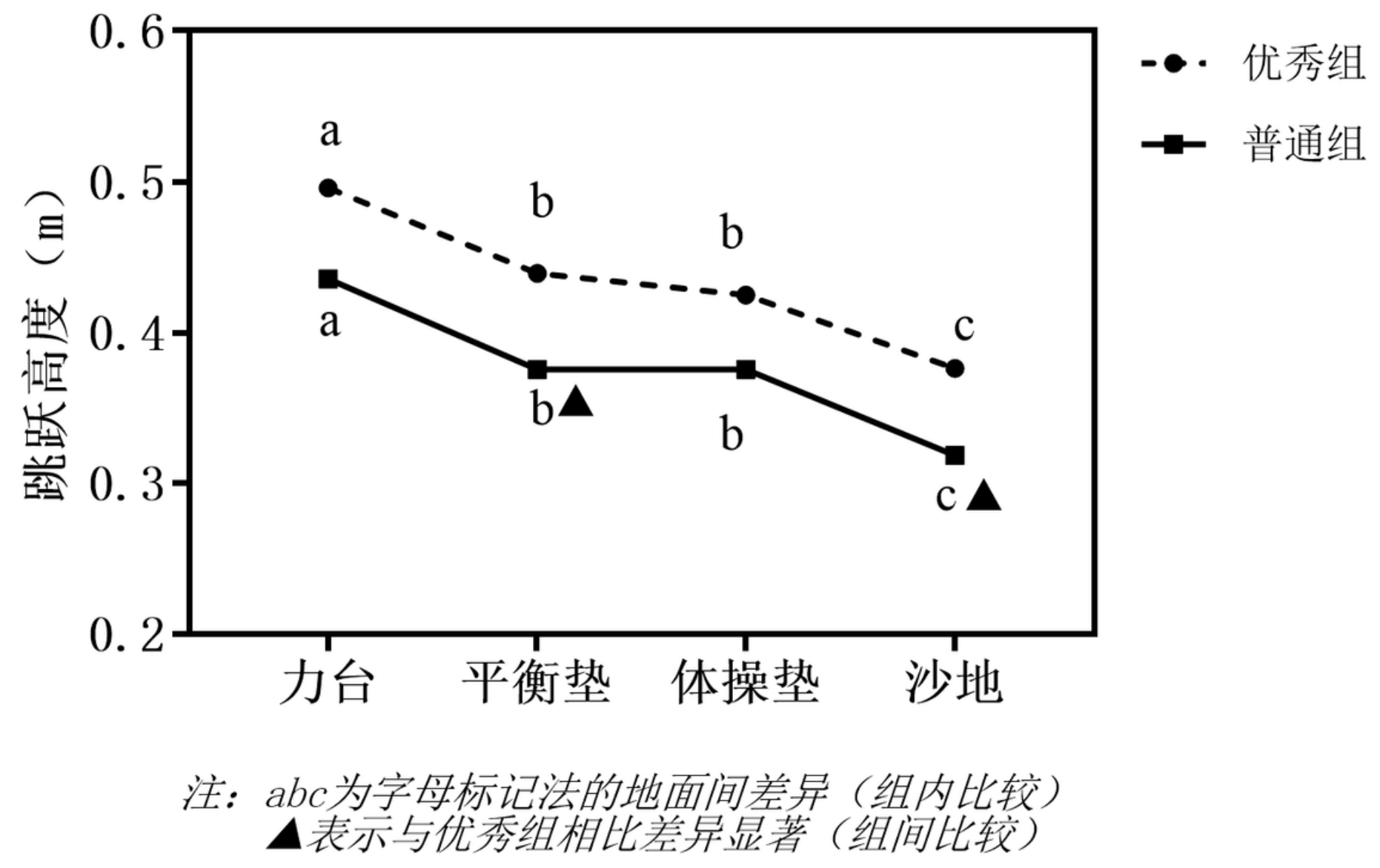

Figure 1

Different terrestrial jump height differences in different levels of athletes 

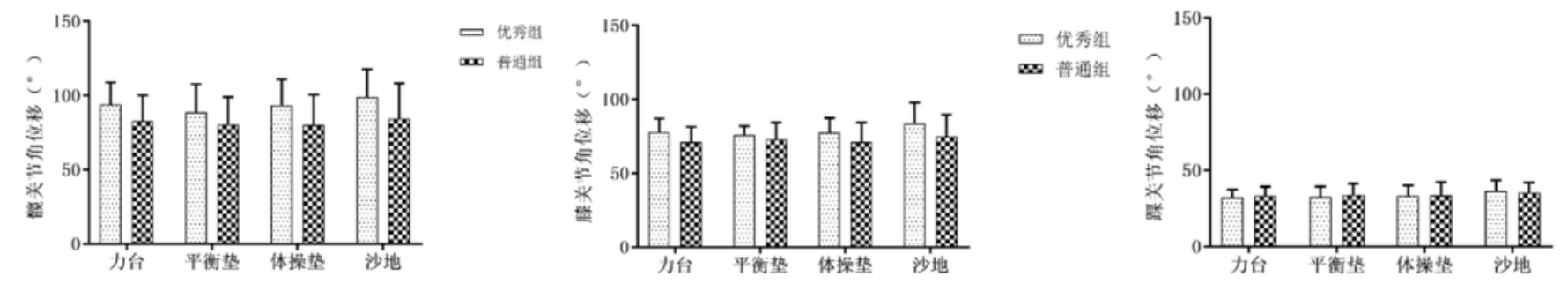

而优悉组

$\mathbf{\infty}$ 粕通组

Figure 2

Different horizontal athletes with different ground hips, knee, ankle buffer angle displacement
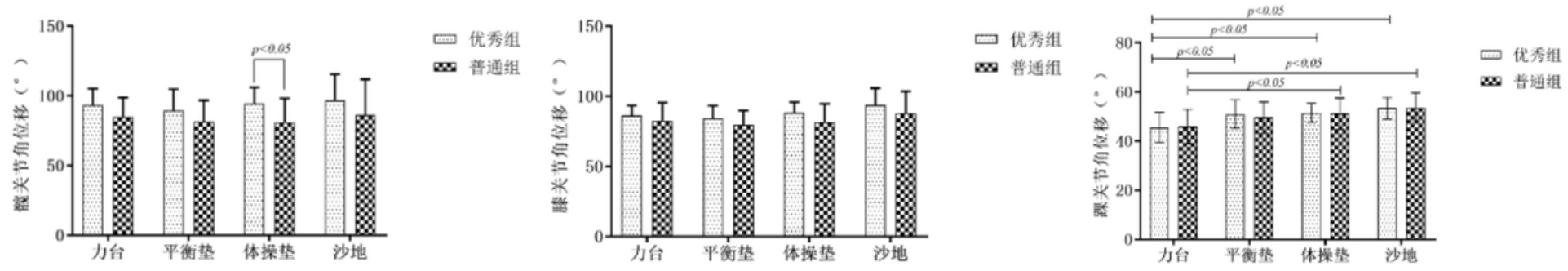

Figure 3

Different horizontal athletes with different ground hips, knee, and ankle
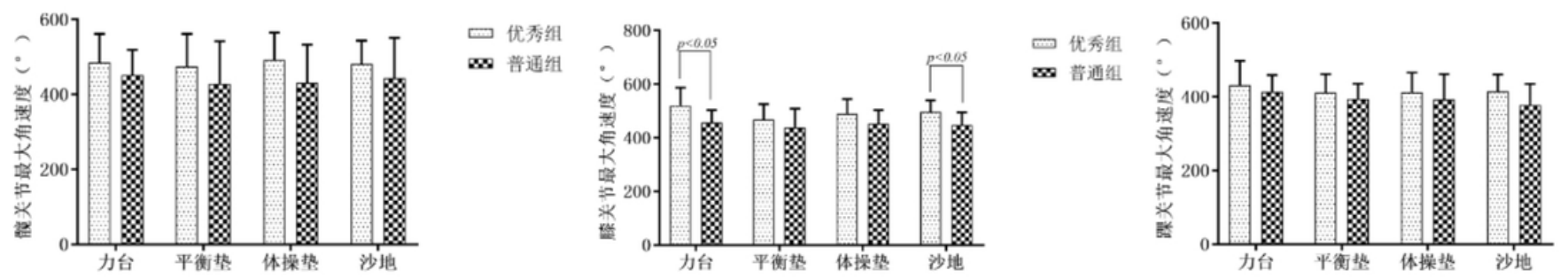

区 位命组 番普通组

Figure 4

Different horizontal athletes with different ground hips, knee, ankle buffer maximum angular velocity
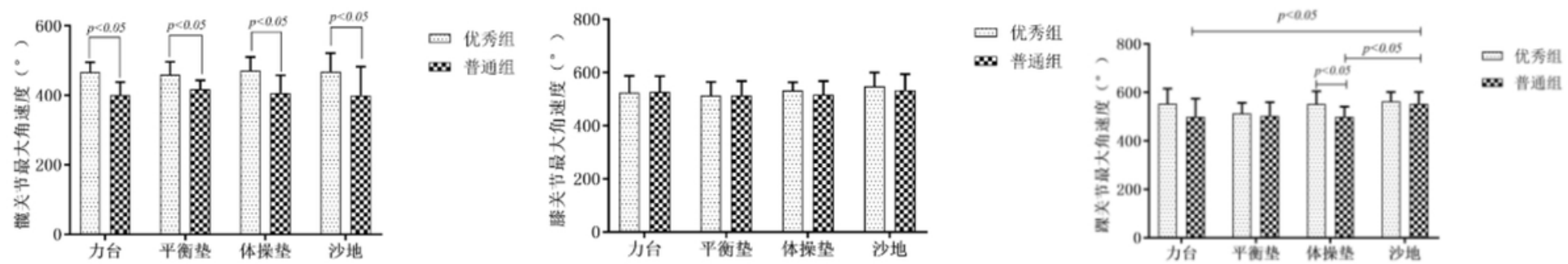

Figure 5

Different horizontal athletes with different ground hips, knee, and ankle 